PROCEEDINGS OF THE

AMERICAN MATHEMATICAL SOCIETY

Volume 140, Number 6, June 2012, Pages 2065-2074

S 0002-9939(2011)11168-7

Article electronically published on October 18, 2011

\title{
SEMI-ALGEBRAIC PARTITION AND BASIS OF BOREL-MOORE HOMOLOGY OF HYPERPLANE ARRANGEMENTS
}

\author{
KO-KI ITO AND MASAHIKO YOSHINAGA \\ (Communicated by Alexander N. Dranishnikov)
}

\begin{abstract}
We describe an explicit semi-algebraic partition for the complement of a real hyperplane arrangement such that each piece is contractible and so that the pieces form a basis of Borel-Moore homology. We also give an explicit correspondence between the de Rham cohomology and the Borel-Moore homology.
\end{abstract}

\section{INTRODUCTION}

Semi-algebraic partitions of algebraic sets have been studied in various fields of mathematics from geometry to computational algebra. The general theory says that any algebraic set has a semi-algebraic triangulation. However, not only triangulations, but also other types of "efficient" decompositions are sometimes useful. The following are motivating examples.

Example 1.1. $\mathbb{C P}^{n}=\mathbb{C}^{n} \sqcup \mathbb{C}^{n-1} \sqcup \cdots \sqcup \mathbb{C}^{1} \sqcup\{p t\}$.

Example 1.2. Consider $\mathbb{C}^{*}=\mathbb{C} \backslash\{0\}$. Put

$$
\begin{aligned}
& S_{0}=\left\{r e^{i \theta} \mid r>0,0<\theta<2 \pi\right\}, \\
& S_{1}=\{z \mid z \in \mathbb{R}, z>0\} .
\end{aligned}
$$

Then $\mathbb{C}^{*}=S_{0} \sqcup S_{1}$.

Both of these decompositions reflect (co)homological structures of the manifolds naturally. More precisely, they are

(i) disjoint unions of contractible semi-algebraic subsets, and

(ii) the closures of the pieces form a basis of Borel-Moore (locally finite) homology $H_{*}^{B M}(X, \mathbb{Z})$ (or ordinary cohomology $H^{*}(X, \mathbb{Z})$ via Poincaré duality).

The purpose of this paper is to generalize Example 1.2 to hyperplane arrangements defined over the real number field $\mathbb{R}$.

There is another reason to expect the existence of such partitions. The complement of a complex hyperplane arrangement is known to be a minimal space [1, 2, 6],

Received by the editors February 10, 2011.

2010 Mathematics Subject Classification. Primary 32S22; Secondary 14N20.

The first author was supported in part by JSPS Grant-in-Aid for Challenging Exploratory Research No. 21654003.

The second author was supported in part by JSPS Grant-in-Aid for Young Scientists (B) No. 20740038.

(C)2011 American Mathematical Society Reverts to public domain 28 years from publication 
that is, a space homotopy equivalent to a finite CW complex with exactly as many $k$-cells as the $k$-th Betti number, for all $k$. If the arrangement is defined over $\mathbb{R}$, then the real structures (e.g., chambers) are related to the topology of the complexified complement $([7,[12)$. With the help of real structures, the minimal CW complex has been explicitly described in [8] and [10]. It is natural to expect that the dual stratification to a minimal CW complex induces a partition satisfying (i) and (ii) above.

\section{Preliminary}

Let $\mathcal{A}=\left\{H_{1}, \ldots, H_{n}\right\}$ be an affine hyperplane arrangement in the real vector space $\mathbb{R}^{\ell}$. Let us fix a defining affine linear form $\alpha_{i}$ in such a way that $H_{i}=\left\{\alpha_{i}=\right.$ $0\}$. Let $L(\mathcal{A})$ be the set of nonempty intersections of elements of $\mathcal{A}$. We denote by $L^{p}(\mathcal{A})$ the set of all $p$-dimensional affine subspaces $X \in L(\mathcal{A})$.

For an affine subspace $X \subset \mathbb{R}^{\ell}$, let us denote by $X_{\mathbb{C}}=X \otimes_{\mathbb{R}} \mathbb{C}$ the complexification. Denote by $\operatorname{ch}(\mathcal{A})$ the set of all chambers and by $\operatorname{M}(\mathcal{A})=\mathbb{C}^{\ell} \backslash \bigcup_{H \in \mathcal{A}} H_{\mathbb{C}}$ the complement to the complexified hyperplanes.

Let $\mathcal{F}$ be a generic flag in $\mathbb{R}^{\ell}$,

$$
\mathcal{F}: \emptyset=\mathcal{F}^{-1} \subset \mathcal{F}^{0} \subset \mathcal{F}^{1} \subset \cdots \subset \mathcal{F}^{\ell}=\mathbb{R}^{\ell},
$$

where each $\mathcal{F}^{q}$ is a generic $q$-dimensional affine subspace, that is, $\operatorname{dim} \mathcal{F}^{q} \cap X=$ $q+\operatorname{dim} X-\ell$ for $X \in L(\mathcal{A})$. Let $\left\{h_{1}, \ldots, h_{\ell}\right\}$ be a system of defining equations of $\mathcal{F}$, that is,

$$
\mathcal{F}^{q}=\left\{h_{q+1}=\cdots=h_{\ell}=0\right\}, \text { for } q=0,1, \ldots, \ell-1,
$$

where each $h_{i}$ is an affine linear form on $\mathbb{R}^{\ell}$. Define

$$
\operatorname{ch}_{\mathcal{F}}^{q}(\mathcal{A})=\left\{C \in \operatorname{ch}(\mathcal{A}) \mid C \cap \mathcal{F}^{q} \neq \emptyset \quad \text { and } \quad C \cap \mathcal{F}^{q-1}=\emptyset\right\} .
$$

We assume that $\mathcal{F}$ satisfies the following:

Assumption 2.1. For $q=0, \ldots, \ell, \mathcal{F}_{>0}^{q}$ denotes

$$
\left\{h_{q+1}=h_{q+2}=\cdots=h_{\ell}=0, h_{q}>0\right\} .
$$

(1) For an arbitrary chamber $C$, if it belongs to $\operatorname{ch}_{\mathcal{F}}^{q}(\mathcal{A})$, then $C \cap \mathcal{F}^{q} \subset \mathcal{F}_{>0}^{q}$.

(2) For any two $X, X^{\prime} \in L(\mathcal{A})$ with $\operatorname{dim} X=\operatorname{dim} X^{\prime}=\ell-q$ (i.e. satisfying $X \cap \mathcal{F}^{q}=\{1 p t\}$ and $\left.X^{\prime} \cap \mathcal{F}^{q}=\{1 p t\}\right)$, if $X \neq X^{\prime}$, then

$$
h_{q}\left(X \cap \mathcal{F}^{q}\right) \neq h_{q}\left(X^{\prime} \cap \mathcal{F}^{q}\right) .
$$

Note that such a flag always exists. Indeed, we first choose a generic hyperplane $\mathcal{F}^{\ell-1}$ in such a way that $\mathcal{F}^{\ell-1}$ does not separate 0 -dimensional intersections $L^{0}(\mathcal{A})$. In a similar fashion, we choose $\mathcal{F}^{\ell-2} \subset \mathcal{F}^{\ell-1}$ inductively.

Let us denote $\mathrm{M}^{q}:=\left(\mathcal{F}_{\mathbb{C}}^{q}\right) \cap \mathrm{M}(\mathcal{A})=\mathcal{F}_{\mathbb{C}}^{q} \backslash \bigcup_{H \in \mathcal{A}} H_{\mathbb{C}}$. We have the following propositions (see [5, 11]).

Proposition 2.1. Let $\mathcal{A}$ be an arrangement and $W^{q}$ a q-dimensional generic subspace. Let $\mathcal{A} \cap W^{q}$ be the arrangement on $W^{q}$ induced by $\mathcal{A}$.

(1) Then $L\left(\mathcal{A} \cap W^{q}\right)$ is isomorphic to $L^{\geq \ell-q}(\mathcal{A})=\{X \in L(\mathcal{A}) \mid \operatorname{dim} X \geq \ell-q\}$ as posets.

(2) Then the natural inclusion $i: \mathrm{M}(\mathcal{A}) \cap W^{q} \hookrightarrow \mathrm{M}(\mathcal{A})$ induces isomorphisms

$$
i_{k}: H_{k}\left(\mathrm{M}(\mathcal{A}) \cap W^{q}, \mathbb{Z}\right) \stackrel{\cong}{\longrightarrow} H_{k}(\mathrm{M}(\mathcal{A}), \mathbb{Z})
$$

for $k=0,1, \ldots, q$. 
Proposition 2.2. Let $\mathcal{A}$ be a real arrangement and $\mathcal{F}$ a generic flag. Then $\left|\operatorname{ch}_{\mathcal{F}}^{q}(\mathcal{A})\right|=b_{q}(\mathrm{M}(\mathcal{A}))$.

Let $X, Y \subset \mathbb{C}^{\ell}$ be subsets. We denote by $\bar{Y}$ the closure of $Y$ in $\mathbb{C}^{\ell}$ (with respect to the classical topology) and $\operatorname{cl}_{X}(Y)=X \cap \bar{Y}$.

For given $C \in \operatorname{ch}_{\mathcal{F}}^{q}(\mathcal{A}), \operatorname{cl}_{\mathcal{F}^{q}}(C)=\bar{C} \cap \mathcal{F}^{q} \subset \mathbb{R}^{\ell}$ is a $q$-dimensional polytope which does not intersect $\mathcal{F}^{q-1}$. By Assumption 2.1, the vertices of $\operatorname{cl}_{\mathcal{F}^{q}}(C)$ have mutually different and positive heights with respect to $h_{q}$ (noting that $\mathcal{F}^{q-1}=\mathcal{F}^{q} \cap\left\{h_{q}=0\right\}$ ). There is a unique vertex $p \in \operatorname{cl}_{\mathcal{F}^{q}}(C)$ at which $\left.h_{q}\right|_{\mathrm{cl}_{\mathcal{F}}(C)}$ attains the minimum. Then by Proposition 2.1 (1), there exists a unique intersection $X_{C} \in L(\mathcal{A})$ satisfying $\{p\}=X_{C} \cap \mathcal{F}^{q}$. (Note that in case $C \in \operatorname{ch}_{\mathcal{F}}^{0}(\mathcal{A})$, we consider $X_{C}=\mathbb{R}^{\ell}$.)

Definition 2.2. Let $X \subset \mathbb{R}^{\ell}$ be an affine subspace. Denote by $\tau(X)$ the linear subspace through the origin which is parallel to $X$ and $\operatorname{dim} \tau(X)=\operatorname{dim} X$, and define

$$
\mathcal{A}_{[X]}:=\{H \in \mathcal{A} \mid \tau(H) \supset \tau(X)\}
$$

to be the set of hyperplanes parallel to $X$. Note that $\mathcal{A}_{X}:=\{H \in \mathcal{A} \mid H \supset X\} \subseteq$ $\mathcal{A}_{[X]}$.

Definition 2.3. Let $C \in \operatorname{ch}_{\mathcal{F}}^{q}(\mathcal{A})$. We denote by $\widetilde{C} \in \operatorname{ch}\left(\mathcal{A}_{\left[X_{C}\right]}\right)$ the unique chamber with $C \subset \widetilde{C}$.

Using this notation, we shall define a partial ordering $\preceq$ in $\operatorname{ch}_{\mathcal{F}}^{q}(\mathcal{A})$.

Definition 2.4. For $C, C^{\prime} \in \operatorname{ch}_{\mathcal{F}}^{q}(\mathcal{A})$, we denote $C \preceq C^{\prime}$ if and only if $C^{\prime} \subset \widetilde{C}$.

The following is easy.

Lemma 2.5. If $C \preceq C^{\prime}$, then $h_{q}\left(X_{C} \cap \mathcal{F}^{q}\right) \leq h_{q}\left(X_{C^{\prime}} \cap \mathcal{F}^{q}\right)$.

\section{Minimal partition}

In this section, we shall introduce the semialgebraic partition.

Definition 3.1. Let $p_{1}, p_{2} \in \mathbb{R}^{\ell}$. The set $\operatorname{Sep}\left(p_{1}, p_{2}\right)$ of separating hyperplanes is defined by

$$
\operatorname{Sep}\left(p_{1}, p_{2}\right):=\left\{H \in \mathcal{A} \mid\left[p_{1}, p_{2}\right] \cap H \neq \emptyset\right\}
$$

where $\left[p_{1}, p_{2}\right]$ is the closed line segment connecting two points $p_{1}$ and $p_{2}$.

Similarly, we also denote by $\operatorname{Sep}\left(C_{1}, C_{2}\right)$ the set of separating hyperplanes of two chambers $C_{1}, C_{2}$.

Lemma 3.2. Let $C, C^{\prime} \in \operatorname{ch}_{\mathcal{F}}^{q}(\mathcal{A})$. If $X_{C}=X_{C^{\prime}}$, then $\operatorname{Sep}\left(C, C^{\prime}\right) \subset \mathcal{A}_{X_{C}}$.

Proof. Let $H \in \operatorname{Sep}\left(C, C^{\prime}\right)$ and choose a defining equation $f$, i.e., $H=\{f=0\}$. Since $H$ separates $C$ and $C^{\prime}$, we may assume $C \subset\{f \geq 0\}$ and $C^{\prime} \subset\{f \leq 0\}$. Hence $X_{C} \cap \mathcal{F}^{q} \subset \operatorname{cl}_{\mathcal{F}^{q}}(C) \subset\{f \geq 0\}$. Similarly, $X_{C^{\prime}} \cap \mathcal{F}^{q} \subset \operatorname{cl}_{\mathcal{F}^{q}}\left(C^{\prime}\right) \subset\{f \leq 0\}$. We have $X_{C} \cap \mathcal{F}^{q} \subset\{f \geq 0\} \cap\{f \leq 0\} \cap \mathcal{F}^{q}=H \cap \mathcal{F}^{q}$. Then the inclusion $X_{C} \subset H$ follows from Proposition 2.1 (1). This means that $H \in \mathcal{A}_{X_{C}}$.

Lemma 3.3. Let $C \in \operatorname{ch}_{\mathcal{F}}^{q}(\mathcal{A})$ and $C^{\prime} \in \operatorname{ch}_{\mathcal{F}}^{q^{\prime}}(\mathcal{A})$.

(1) If $C^{\prime} \subset \widetilde{C}$, then either $C \preceq C^{\prime}$ (with $q=q^{\prime}$ ) or $q<q^{\prime}$.

(2) If $q=q^{\prime}$ and $h_{q}\left(X_{C} \cap \mathcal{F}^{q}\right)<h_{q}\left(X_{C^{\prime}} \cap \mathcal{F}^{q}\right)$, then $\mathcal{A}_{X_{C^{\prime}}} \cap \operatorname{Sep}\left(C, C^{\prime}\right) \neq \emptyset$.

(3) If $q<q^{\prime}$, then $\mathcal{A}_{X_{C^{\prime}}} \cap \operatorname{Sep}\left(C, C^{\prime}\right) \neq \emptyset$. 
Proof. (1) First note that $\widetilde{C} \cap \mathcal{F}^{q-1}=\left(\widetilde{C} \cap \mathcal{F}^{q}\right) \cap \mathcal{F}^{q-1}$. Since $\left.h_{q}\right|_{\widetilde{C} \cap \mathcal{F}^{q}}$ attains the minimum at $X_{C} \cap \mathcal{F}^{q},\left.h_{q}\right|_{\widetilde{C} \cap \mathcal{F}^{q}}>0$. Hence $C^{\prime} \cap \mathcal{F}^{q-1}=\emptyset$ and we have $q^{\prime} \geq q$. The assertions thus follow from Definition 2.4.

(2) Suppose that $\mathcal{A}_{X_{C^{\prime}}} \cap \operatorname{Sep}\left(C, C^{\prime}\right)=\emptyset$. Then $C$ and $C^{\prime}$ are contained in the same chamber $D \in \operatorname{ch}\left(\mathcal{A}_{X_{C^{\prime}}}\right)$ of $\mathcal{A}_{X_{C^{\prime}}}$. Since $\left.h_{q}\right|_{\mathrm{cl}_{\mathcal{F}}(D)}$ attains the minimum at $X_{C^{\prime}} \cap \mathcal{F}^{q}$, we have $h_{q}\left(X_{C} \cap \mathcal{F}^{q}\right) \geq h_{q}\left(X_{C^{\prime}} \cap \mathcal{F}^{q}\right)$. This contradicts the assumption.

(3) Suppose that $\mathcal{A}_{X_{C^{\prime}}} \cap \operatorname{Sep}\left(C, C^{\prime}\right)=\emptyset$. Then $C$ and $C^{\prime}$ are contained in the same chamber $D \in \operatorname{ch}\left(\mathcal{A}_{X_{C^{\prime}}}\right)$ of $\mathcal{A}_{X_{C^{\prime}}}$. Obviously $D \cap \mathcal{F}^{q^{\prime}-1}=\emptyset$ and hence $C \cap \mathcal{F}^{q}=\emptyset$. This contradicts $C \in \operatorname{ch}_{\mathcal{F}}^{q}(\mathcal{A})$.

From now on we fix a base point $p_{C} \in C \cap \mathcal{F}^{q}$ for each $C \in \operatorname{ch}_{\mathcal{F}}^{q}(\mathcal{A})$. It is easily seen that the constructions below do not depend on the choice of $p_{C}$.

We can identify $\mathbb{C}^{\ell}$ with the tangent bundle $T \mathbb{R}^{\ell} \cong \mathbb{R}^{\ell} \times \mathbb{R}^{\ell}$ by

$$
\begin{aligned}
\mathbb{R}^{\ell} \times \mathbb{R}^{\ell} & \longrightarrow \mathbb{C}^{\ell} \\
(x, v) & \longmapsto x+\sqrt{-1} v .
\end{aligned}
$$

We also denote $x$ by $\operatorname{Re}(x+\sqrt{-1} v)$.

Now we introduce the main object of this paper.

Definition 3.4. For a chamber $C \in \operatorname{ch}(\mathcal{A})$, we define

$$
S(C)=\left\{\begin{array}{l|l}
x+\sqrt{-1} v \in \mathbb{C}^{\ell} & \begin{array}{l}
v \in \tau\left(X_{C}\right), x \in \mathbb{R}^{\ell} \text { and } \\
v \notin \tau(H), \text { for } H \in \operatorname{Sep}\left(p_{C}, x\right)
\end{array}
\end{array}\right\} .
$$

If $C \in \operatorname{ch}_{\mathcal{F}}^{q}(\mathcal{A}), S(C)$ is an open subset of $\mathbb{R}^{\ell} \times \tau\left(X_{C}\right)$, hence a real $(2 \ell-q)$ dimensional manifold.

Example 3.5. Let $H=\{0\} \subset \mathbb{R}$ and the arrangement $\mathcal{A}=\{H\}$. Fix a generic flag $\mathcal{F}^{0}=\{-1\}$. There are two chambers $C_{0}=\mathbb{R}_{<0}$ and $C_{1}=\mathbb{R}_{>0}$. Then $\operatorname{ch}_{\mathcal{F}}^{0}(\mathcal{A})=\left\{C_{0}\right\}$ and $\operatorname{ch}_{\mathcal{F}}^{1}(\mathcal{A})=\left\{C_{1}\right\}$. Then $S\left(C_{0}\right)=S_{0}$ and $S\left(C_{1}\right)=S_{1}$ as defined in Example 1.2

Example 3.6. Let $\mathcal{A}=\left\{H_{1}, H_{2}, H_{3}\right\}$ be an arrangement of lines on $\mathbb{R}^{2}$ and fix a generic flag $\mathcal{F}^{\bullet}$ as in Figure 1. Then $\operatorname{ch}_{\mathcal{F}}^{0}(\mathcal{A})=\left\{C_{0}\right\}, \operatorname{ch}_{\mathcal{F}}^{1}(\mathcal{A})=\left\{C_{1}, C_{2}, C_{3}\right\}$, $\operatorname{ch}_{\mathcal{F}}^{2}(\mathcal{A})=\left\{C_{4}, C_{5}\right\}$. By definition, we also have $X_{C_{0}}=\mathbb{R}^{2}, X_{C_{1}}=H_{1}, X_{C_{2}}=$ $H_{2}, X_{C_{3}}=H_{3}$, and $X_{C_{4}}=H_{1} \cap H_{3}, X_{C_{5}}=H_{1} \cap H_{2}, \mathcal{A}_{\left[X_{C_{0}}\right]}=\emptyset, \mathcal{A}_{\left[X_{C_{1}}\right]}=\left\{H_{1}\right\}$, $\mathcal{A}_{\left[X_{C_{2}}\right]}=\mathcal{A}_{\left[X_{C_{3}}\right]}=\left\{H_{2}, H_{3}\right\}, \mathcal{A}_{\left[X_{C_{4}}\right]}=\mathcal{A}_{\left[X_{C_{5}}\right]}=\mathcal{A}, \widetilde{C_{0}}=\mathbb{R}^{2}, \widetilde{C_{1}}=C_{1} \cup C_{2} \cup C_{3}$, $\widetilde{C_{2}}=C_{2} \cup C_{5}, \widetilde{C_{3}}=C_{3} \cup C_{4}, \widetilde{C_{4}}=C_{4}, \widetilde{C_{5}}=C_{5}$. Using these data, we can describe $S(C)$. For example, $S\left(C_{4}\right)=C_{4}, S\left(C_{5}\right)=C_{5}$. Other pieces are shown in Figure 1 . (In the figure, a dotted line indicates the direction to which $v$ cannot be directed.)

Remark 3.7. The above example shows that our decomposition is not always a Whitney stratification. Indeed, $\operatorname{dim} S\left(C_{1}\right)=3$ and $\operatorname{cl}_{\mathrm{M}(\mathcal{A})}\left(S\left(C_{1}\right)\right) \backslash S\left(C_{1}\right)=C_{2} \cup C_{3}$. However the subset $C_{2} \cup C_{3}$ is not a union of our 2-dimensional components $S\left(C_{4}\right)$ and $S\left(C_{5}\right)$.

Lemma 3.8. The real part $\operatorname{Re} S(C)=\left\{\operatorname{Re}(z) \in \mathbb{R}^{\ell} \mid z \in S(C)\right\}$ of $S(C)$ coincides with $\widetilde{C}$.

Proof. Assume $x \in \operatorname{Re} S(C)$. Then there exists $v \in \mathbb{R}^{\ell}$ such that $x+\sqrt{-1} v \in S(C)$. Let $H \in \mathcal{A}_{\left[X_{C}\right]}$. By definition, $v \in \tau\left(X_{C}\right) \subset \tau(H)$, and $H \notin \operatorname{Sep}\left(p_{C}, x\right)$. Hence 

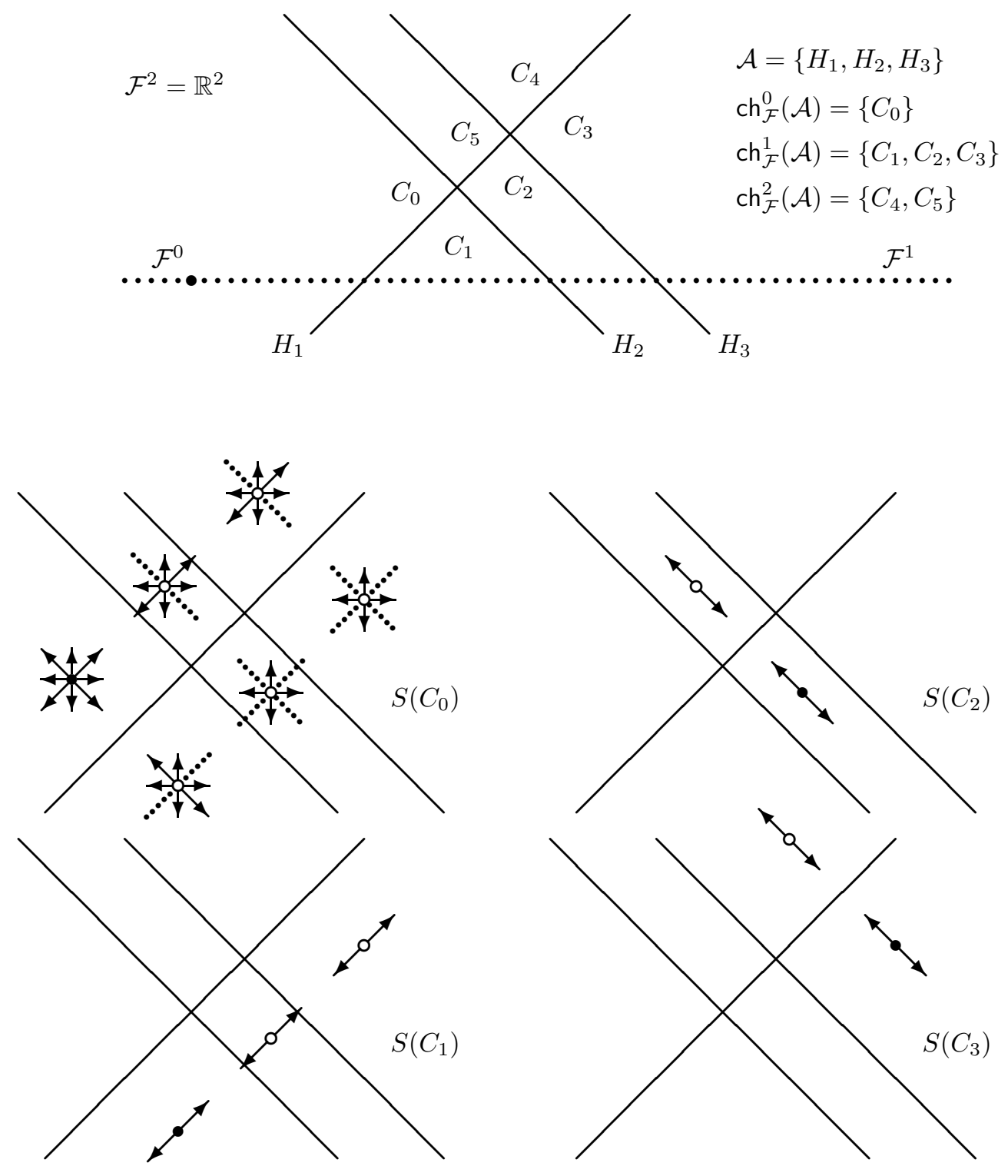

Figure 1. Example 3.6

$p_{C}$ and $x$ are not separated by any hyperplane $H$ belonging to $\mathcal{A}_{\left[X_{C}\right]}$; we have $\operatorname{Re} S(C) \subset \widetilde{C}$.

Conversely, assume $x \in \widetilde{C}$. Since $x$ and $p_{C}$ are contained in the same chamber of $\mathcal{A}_{\left[X_{C}\right]}$, we have $\operatorname{Sep}\left(p_{C}, x\right) \cap \mathcal{A}_{\left[X_{C}\right]}=\emptyset$. Choose $v \in \tau\left(X_{C}\right) \backslash \bigcup_{H \in \mathcal{A} \backslash \mathcal{A}_{\left[X_{C}\right]}} \tau(H)$. Then $x+\sqrt{-1} v \in S(C)$.

Lemma 3.9. If $C \in \operatorname{ch}_{\mathcal{F}}^{q}(\mathcal{A})$, then $S(C)$ is a contractible $(2 \ell-q)$-dimensional manifold. 
Proof. Let us prove that $S(C)$ is star-shaped. For a point $x+\sqrt{-1} v \in S(C)$, consider the path $p(t)$ with parameter $0 \leq t \leq 1$ :

$$
p(t)=(1-t) p_{C}+t(x+\sqrt{-1} v)=\left((1-t) p_{C}+t x\right)+\sqrt{-1} t v .
$$

We have $p(1)=x+\sqrt{-1} v$ and $p(0)=p_{C}$. It suffices to prove that $p(t) \in \mathrm{M}(\mathcal{A})$ for $0 \leq t \leq 1$. If $\operatorname{Re} p(t)=(1-t) p_{C}+t x \notin H$, then obviously we have $p(t) \notin H_{\mathbb{C}}$. Suppose $(1-t) p_{C}+t x \in H$ for some $t$ with $0<t \leq 1$. Then, by assumption, $H \in \operatorname{Sep}\left(p_{C}, x\right)$. By the definition of $S(C), v$ is transverse to $H$, so is $t v$, which means $p(t) \in \mathrm{M}(\mathcal{A})$. Hence $S(C)$ is star-shaped.

Now we have the following:

Theorem 3.10. The complement $\mathrm{M}(\mathcal{A})$ of $\mathcal{A}$ is a disjoint union of $S(C), C \in$ $\operatorname{ch}(\mathcal{A})$, namely,

$$
\mathrm{M}(\mathcal{A})=\bigsqcup_{C \in \operatorname{ch}(\mathcal{A})} S(C) .
$$

Proof. First we prove that $S(C) \cap S\left(C^{\prime}\right)=\emptyset$ when $C \neq C^{\prime}$. Suppose this is not the case. Then there exists a point $x+\sqrt{-1} v \in S(C) \cap S\left(C^{\prime}\right)$.

(a) If both $C, C^{\prime}$ are in $\operatorname{ch}_{\mathcal{F}}^{q}(\mathcal{A})$ and $X_{C} \neq X_{C^{\prime}}$, then we may assume $h_{q}\left(X_{C} \cap\right.$ $\left.\mathcal{F}^{q}\right)<h_{q}\left(X_{C^{\prime}} \cap \mathcal{F}^{q}\right)$. From Lemma 2.5 we have $C^{\prime} \npreceq C$. By Lemma $3.3(2)$, there exists $H \in \mathcal{A}_{X_{C^{\prime}}} \cap \operatorname{Sep}\left(C, C^{\prime}\right)$. By definition of $S\left(C^{\prime}\right), x+\sqrt{-1} v \in$ $S\left(C^{\prime}\right)$ implies that

$$
\mathcal{A}_{X_{C^{\prime}}} \cap \operatorname{Sep}\left(x, p_{C^{\prime}}\right)=\emptyset
$$

and

$$
v \in \tau\left(X_{C^{\prime}}\right) .
$$

It follows from (3.1) that $x$ and $p_{C}$ are separated by $H$, and from (3.2) that $v \in \tau(H)$. (Note that $\tau\left(X_{C^{\prime}}\right) \subset \tau(H)$.) Then we have $x+\sqrt{-1} v \notin S(C)$, which contradicts the assumption; this concludes $S(C) \cap S\left(C^{\prime}\right)=\emptyset$.

(b) Next we consider the case that $C$ and $C^{\prime}$ are in $\operatorname{ch}_{\mathcal{F}}^{q}(\mathcal{A})$ and $X_{C}=X_{C^{\prime}}$. By Lemma 3.2. $C$ and $C^{\prime}$ are separated by a hyperplane $H \in \mathcal{A}_{X_{C}}$. This implies that $H$ separates $\widetilde{C}$ and $\widetilde{C^{\prime}}$. By Lemma 3.8 we have $\operatorname{Re} S(C) \cap \operatorname{Re} S\left(C^{\prime}\right)=\emptyset$.

(c) Finally, we consider the case $C \in \operatorname{ch}_{\mathcal{F}}^{q}(\mathcal{A})$ and $C^{\prime} \in \operatorname{ch}_{\mathcal{F}}^{q^{\prime}}(\mathcal{A})$, with $q<q^{\prime}$. Then again by Lemma 3.3(3), there exists a hyperplane $H \in \mathcal{A}_{X_{C^{\prime}}}$ separating $C$ and $C^{\prime}$. As in the case (a), we obtain $x+\sqrt{-1} v \notin S(C)$. Therefore $S(C) \cap S\left(C^{\prime}\right)=\emptyset$.

Next we prove that

$$
\mathrm{M}(\mathcal{A})=\bigcup_{C \in \operatorname{ch}(\mathcal{A})} S(C)
$$

Let $x+\sqrt{-1} v \in \mathrm{M}(\mathcal{A})$. Recall that $\mathcal{A}_{[v]}$ is the set of all hyperplanes parallel to $v$, namely, $\mathcal{A}_{[v]}=\{H \in \mathcal{A} \mid \tau(H) \ni v\}$. Since $v$ is parallel to hyperplanes in $\mathcal{A}_{[v]}, x$ is not contained in $H \in \mathcal{A}_{[v]}$. We can choose a chamber $D \in \operatorname{ch}\left(\mathcal{A}_{[v]}\right)$ such that $x \in D$. Let $q=\min \left\{i \mid D \cap \mathcal{F}^{i} \neq \emptyset\right\}$. Since the closure $\operatorname{cl}_{\mathcal{F}^{q}}(D)$ is a convex polytope in $\mathcal{F}^{q}$ which does not intersect with $\mathcal{F}^{q-1}$, there exists a unique point $p \in \operatorname{cl}_{\mathcal{F}^{q}}(D)$ of the minimum with respect to $h_{q}$. We can choose $X \in L(\mathcal{A})$ such that $p=X \cap \mathcal{F}^{q}$. 
Note that $X=\bigcap_{H \in \mathcal{A}_{p}} H$ and then $v \in \tau(X)$. There exists $C \in \operatorname{ch}_{\mathcal{F}}^{q}(\mathcal{A})$ satisfying $X_{C}=X$ and $C \subset D$. We prove that

$$
x+\sqrt{-1} v \in S(C) .
$$

It is enough to prove that $v \notin \tau(H)$ for $H \in \operatorname{Sep}\left(x, p_{C}\right)$. Note that $x$ and $p_{C}$ are contained in the same chamber $D \in \operatorname{ch}\left(\mathcal{A}_{[v]}\right)$. Hence if $H \in \operatorname{Sep}\left(x, p_{C}\right)$, then $H \notin \mathcal{A}_{[v]}$. By definition of $\mathcal{A}_{[v]}, v \notin \tau(H)$. Therefore $v$ is transverse to $H$, which means that $x+\sqrt{-1} v \in S(C)$.

\section{Basis of BM-HOMOLOGY}

In this section, we shall prove that the closures $\left\{\operatorname{cl}_{\mathrm{M}(\mathcal{A})}(S(C))\right\}_{C \in \operatorname{ch}_{\mathcal{F}}^{q}(\mathcal{A})}$ form a basis of $H_{2 \ell-q}^{B M}(\mathrm{M}(\mathcal{A}), \mathbb{Z})$. In 4 4.1, we determine orientations on our spaces. In 4.2 we recall the constructions of a basis $\left\{\left[\sigma_{C}\right] \mid C \in \operatorname{ch}_{\mathcal{F}}^{q}(\mathcal{A})\right\}$ of $H_{q}(\mathrm{M}(\mathcal{A}), \mathbb{Z})$ from [10]. By computing intersection numbers of $\mathrm{cl}_{\mathrm{M}}(S(C))$ and $\left[\sigma_{C^{\prime}}\right]$, in 4.2, we prove the main result.

4.1. Orientations. In this section, we shall define orientations for $\mathcal{F}^{q}, X_{C}$ and $S(C)$ by choosing an ordered basis of the tangent spaces. (See chapter 3 of [3] for generalities of orientations and intersections of manifolds.)

Recall that the subspace $\mathcal{F}^{q}$ is defined by $\left\{x \in \mathbb{R}^{\ell} \mid h_{q+1}(x)=\cdots=h_{\ell}(x)=0\right\}$, where $h_{i}(i=1, \ldots, \ell)$ are linear forms. Hence $\left(h_{1}, \ldots, h_{q}\right)$ forms a coordinate of the space $\mathcal{F}^{q}$. We consider the orientation defined by the ordered basis $\left(\partial_{h_{1}}, \ldots, \partial_{h_{q}}\right)$ of $T_{x} \mathcal{F}^{q}=\tau\left(\mathcal{F}^{q}\right)$. In particular, the orientation of $\mathbb{R}^{\ell}$ is determined by the ordered basis $\left(\partial_{h_{1}}, \ldots, \partial_{h_{\ell}}\right)$. If $C$ belongs to $\operatorname{ch}_{\mathcal{F}}^{q}(\mathcal{A})$, then $X_{C}$ is an affine subspace complemental to $\mathcal{F}^{q}$. So $\left(h_{q+1}, \ldots, h_{\ell}\right)$ forms a coordinate of $X_{C}$, and we consider the orientation determined by the dual basis $\left(\partial_{h_{q+1}}, \ldots, \partial_{h_{\ell}}\right)$ with an order. Note that the intersection number $\mathcal{F}^{q} \cdot X_{C}=(-1)^{q(\ell-q)} \cdot X_{C} \cdot \mathcal{F}^{q}$ equals +1 .

Next we consider the orientation of $S(C)$. By definition, the tangent space of $S(C)$ at $p_{C}$ is expressed as

$$
T_{p_{C}} S(C) \simeq T_{p_{C}} C \oplus \sqrt{-1} \cdot \tau\left(X_{C}\right) .
$$

Thus we define the orientation by $\left(\partial_{h_{1}}, \partial_{h_{2}}, \ldots, \partial_{h_{\ell}}, \sqrt{-1} \partial_{h_{q+1}}, \ldots, \sqrt{-1} \partial_{h_{\ell}}\right)$. The case $q=0$ defines an orientation on $\mathbb{C}^{\ell}$ by $\left(\partial_{h_{1}}, \ldots, \partial_{h_{\ell}}, \sqrt{-1} \partial_{h_{1}}, \ldots, \sqrt{-1} \partial_{h_{\ell}}\right)$. We should note that this orientation is different from the usual one defined by $\left(\partial_{h_{1}}, \sqrt{-1} \partial_{h_{1}}, \partial_{h_{2}}, \sqrt{-1} \partial_{h_{2}}, \ldots, \partial_{h_{\ell}}, \sqrt{-1} \partial_{h_{\ell}}\right)$.

The rest will be used in $\$ 5$, Let $I=\left\{i_{1}, \ldots, i_{q}\right\} \subset\{1, \ldots, n\}$ be an ordered subset of $q$ indices, $\mathcal{A}(I):=\left\{H_{i_{1}}, \ldots, H_{i_{q}}\right\}$ be a subarrangement consisting of $q$ hyperplanes. Assume $H_{i_{1}}, \ldots, H_{i_{q}}$ are independent, that is, $d \alpha_{i_{1}} \wedge \cdots \wedge d \alpha_{i_{\ell}} \neq 0$ or equivalently the intersection $X(I):=H_{i_{1}} \cap \cdots \cap H_{i_{q}}$ is a nonempty subspace of codimension $q$.

Definition-Lemma 4.1. The set of chambers $\operatorname{ch}(\mathcal{A}(I))$ consists of $2^{q}$ chambers. There is a unique chamber, denoted by $C_{0}(I) \in \operatorname{ch}(\mathcal{A}(I))$, which satisfies $C_{0}(I) \cap$ $\mathcal{F}^{q-1}=\emptyset$.

Proof. The Poincaré polynomial of $\mathbb{C}^{\ell} \backslash \bigcup_{i \in I} H_{i, \mathbb{C}}$ is $(1+t)^{q}$. In particular, $b_{q}=1$. Hence by Proposition 2.2, $\left|\operatorname{ch}_{\mathcal{F}}^{q}(\mathcal{A}(I))\right|=1$.

Choose a normal vector $w_{i_{k}} \perp H_{i_{k}}$ for each $H_{i_{k}}$ such that $C_{0}(I)$ is contained in the half-space $H_{i_{k}}+\mathbb{R}_{>0} \cdot w_{i_{k}}$. Suppose $H_{i_{1}}, \ldots, H_{i_{q}}$ are independent (i.e., the 
intersection $X(I)=H_{i_{1}} \cap \cdots \cap H_{i_{q}}$ has codimension $q$ with $\left.q \leq \ell\right)$. Since $\mathcal{F}^{q}$ is generic, $\mathcal{F}^{q} \cap X(I)$ is 0 -dimensional. Thus by the identification $\mathbb{R}^{\ell} / X(I) \simeq \mathcal{F}^{q}$, the normal vectors $w_{i_{1}}, \ldots, w_{i_{q}}$ induce a basis of $\mathcal{F}^{q}$.

Definition 4.2. For an ordered $q$-tuple $I=\left\{i_{1}, \ldots, i_{q}\right\} \subset\{1, \ldots, n\}$, define $\varepsilon(I)$ by

$$
\varepsilon(I)= \begin{cases}0 & \text { if } H_{i_{1}}, \ldots, H_{i_{q}} \text { are dependent, } \\ 1 & \text { if }\left(w_{i_{1}}, \ldots, w_{i_{q}}\right) \text { induces a positive basis of } \mathcal{F}^{q} \\ -1 & \text { if }\left(w_{i_{1}}, \ldots, w_{i_{q}}\right) \text { induces a negative basis of } \mathcal{F}^{q}\end{cases}
$$

4.2. Minimal CW-decomposition. Here we recall results from [10, §5.2]. For each $C \in \operatorname{ch}_{\mathcal{F}}^{q}(\mathcal{A})$, there exists a continuous map, unique up to homotopy,

$$
\sigma_{C}:\left(D^{q}, \partial D^{q}\right) \longrightarrow\left(\mathrm{M}^{q}, \mathrm{M}^{q-1}\right),
$$

from the $q$-dimensional disk to the complement $\mathrm{M}^{q}=\mathrm{M}(\mathcal{A}) \cap \mathcal{F}_{\mathbb{C}}^{q}$ such that

(Transversality) $\sigma_{C}(0)=p_{C} \in C \cap \mathcal{F}^{q}$, and $\sigma_{C}\left(D^{q}\right)$ intersects $C \cap$

$\mathcal{F}^{q}$ transversally in $\mathcal{F}_{\mathbb{C}}^{q}$ at the point; $\sigma_{C}\left(D^{q}\right) \pitchfork C=\left\{p_{C}\right\}$, and

(Non-intersecting) $\sigma_{C}\left(D^{q}\right) \cap C^{\prime}=\emptyset$ for $C^{\prime} \in \operatorname{ch}_{\mathcal{F}}^{q}(\mathcal{A}) \backslash\{C\}$.

These properties guarantee the following homotopy equivalence ([10, 4.3.1]):

$$
\mathrm{M}^{q} \simeq \mathrm{M}^{q-1} \cup_{\left(\partial \sigma_{C}\right)}\left(\bigsqcup_{C \in \operatorname{ch}_{\mathcal{F}}^{q}(\mathcal{A})} D^{q}\right)
$$

where the right-hand side is obtained by attaching $q$-dimensional disks to $\mathrm{M}^{q-1}$ along $\partial \sigma_{C}: \partial D^{q} \rightarrow \mathrm{M}^{q-1}$ for $C \in \operatorname{ch}_{\mathcal{F}}^{q}(\mathcal{A})$.

Recall that $T_{p_{C}} \mathrm{M}^{q} \simeq \tau\left(\mathcal{F}^{q}\right) \oplus \sqrt{-1} \cdot \tau\left(\mathcal{F}^{q}\right)$. We introduce an orientation on $\sigma_{C}$ by identifying $T_{p_{C}} \sigma_{C}\left(D^{q}\right)$ with $\sqrt{-1} \cdot \tau\left(\mathcal{F}^{q}\right)$, equivalently, by an ordered basis $\left(\sqrt{-1} \partial_{h_{1}}, \ldots, \sqrt{-1} \partial_{h_{q}}\right)$.

Proposition $4.1([10])$. (1) $\left[\sigma_{C}\right] \in H_{q}\left(\mathrm{M}^{q}, \mathrm{M}^{q-1} ; \mathbb{Z}\right),\left(C \in \mathrm{ch}_{\mathcal{F}}^{q}(\mathcal{A})\right)$ forms a basis.

(2) $H_{q}\left(\mathrm{M}^{q}, \mathbb{Z}\right) \simeq H_{q}\left(\mathrm{M}^{q}, \mathrm{M}^{q-1} ; \mathbb{Z}\right) \simeq H_{q}(\mathrm{M}(\mathcal{A}), \mathbb{Z})$.

We construct the basis of $H_{2 \ell-q}^{B M}(\mathrm{M}(\mathcal{A}), \mathbb{Z})$. Let $C \in \operatorname{ch}_{\mathcal{F}}^{q}(\mathcal{A})$. Lemma 3.8 indicates that

$$
\operatorname{cl}_{\mathrm{M}(\mathcal{A})}(S(C))=\left(\widetilde{C} \times \sqrt{-1} \cdot \tau\left(X_{C}\right)\right) \cap \mathrm{M}(\mathcal{A}),
$$

which is a closed oriented $(2 \ell-q)$-dimensional submanifold of $\mathrm{M}(\mathcal{A})$ because $\operatorname{dim} X_{C}$ $=\ell-q$. The closed submanifold $\operatorname{cl}_{\mathrm{M}(\mathcal{A})}(S(C))$ determines a cycle $\left[\operatorname{cl}_{\mathrm{M}(\mathcal{A})}(S(C))\right] \in$ $H_{2 \ell-q}^{B M}(\mathrm{M}(\mathcal{A}), \mathbb{Z})$.

Theorem 4.3. The classes $\left\{\left[\operatorname{cl}_{\mathrm{M}(\mathcal{A})}(S(C))\right]\right\}_{C \in \mathrm{ch}_{\mathcal{F}}^{q}(\mathcal{A})}$ form a basis of the $(2 \ell-q)$-th Borel-Moore homology group $H_{2 \ell-q}^{B M}(\mathrm{M}(\mathcal{A}), \mathbb{Z})$.

Proof. We compute the intersection number of $\left[\operatorname{cl}_{\mathrm{M}(\mathcal{A})}(S(C))\right] \in H_{2 \ell-q}^{B M}(\mathrm{M}(\mathcal{A}))$ and $\left[\sigma\left(C^{\prime}\right)\right] \in H_{q}(\mathrm{M}(\mathcal{A}))$, and show that the intersection matrix

$$
I\left(\left[\operatorname{cl}_{\mathrm{M}(\mathcal{A})}(S(C))\right],\left[\sigma\left(C^{\prime}\right)\right]\right)_{C, C^{\prime} \in \operatorname{ch}_{\mathcal{F}}^{q}(\mathcal{A})}
$$

is a triangular matrix with each diagonal entry $(-1)^{q(\ell-q)}$.

We fix an ordering on $\left\{C_{1}, \ldots, C_{b}\right\}=\operatorname{ch}_{\mathcal{F}}^{q}(\mathcal{A})$ in such a way that $C_{i} \preceq C_{j} \Longrightarrow i<$ $j$ (e.g. choose an ordering with $\left.h_{q}\left(X_{C_{1}} \cap \mathcal{F}^{q}\right) \leq h_{q}\left(X_{C_{2}} \cap \mathcal{F}^{q}\right) \leq \cdots \leq h_{q}\left(X_{C_{b}} \cap \mathcal{F}^{q}\right)\right)$. 
Since $\mathcal{F}^{q}$ and $X_{C}$ are mutually complementary in $\mathbb{R}^{\ell}$, the tangent space $T_{p_{C}} \mathbb{C}^{\ell}$ can be expressed as

$$
T_{p_{C}} \mathbb{C}^{\ell}=T_{p_{C}} \mathbb{R}^{\ell} \oplus \sqrt{-1} \cdot T_{p_{C}} \mathcal{F}^{q} \oplus \sqrt{-1} \cdot \tau\left(X_{C}\right) .
$$

The above-mentioned properties and (4.2) imply that $\mathrm{cl}_{\mathrm{M}(\mathcal{A})}(S(C))$ intersects transversally to $\sigma_{C^{\prime}}$ if and only if $p_{C^{\prime}} \in \widetilde{C}$. In fact, we have $T_{p_{C}} \operatorname{cl}_{\mathrm{M}(\mathcal{A})}(S(C))=$ $\mathbb{R}^{\ell} \oplus \sqrt{-1} \cdot \tau\left(X_{C}\right)$ and $T_{p_{C^{\prime}}} \sigma_{C^{\prime}}\left(D^{q}\right)=\sqrt{-1} \cdot T_{p_{C^{\prime}}} \mathcal{F}^{q}$, which implies the transversality and its intersection number is $(-1)^{q(\ell-q)}$.

\section{Relations with OS-type Generators}

As is mentioned in $\mathbb{1}$ there is a canonical isomorphism $\varphi: H^{q}(\mathrm{M}(\mathcal{A}), \mathbb{Z}) \stackrel{\cong}{\longrightarrow}$ $H_{2 \ell-q}^{B M}(\mathrm{M}(\mathcal{A}), \mathbb{Z})$ between cohomology and Borel-Moore homology of $\mathrm{M}(\mathcal{A})$. In this section, we describe $\varphi$ explicitly by using the basis introduced in the previous sections.

First note that both $H_{2 \ell-q}^{B M}(\mathrm{M}(\mathcal{A}), \mathbb{Z})$ and $H^{q}(\mathrm{M}(\mathcal{A}), \mathbb{Z})$ are dual to the homology group $H_{q}(\mathrm{M}(\mathcal{A}), \mathbb{Z})$. The pairing $H_{2 \ell-q}^{B M}(\mathrm{M}(\mathcal{A}), \mathbb{Z}) \times H_{q}(\mathrm{M}(\mathcal{A}), \mathbb{Z}) \rightarrow \mathbb{Z}$ is defined by the intersection $I(\cdot, \cdot)$, and $H^{q}(\mathrm{M}(\mathcal{A}), \mathbb{Z}) \times H_{q}(\mathrm{M}(\mathcal{A}), \mathbb{Z}) \rightarrow \mathbb{Z}$ is defined by the cap product $\cap$ (or the integration if we consider de Rham cohomology).

The structure of the cohomology ring $H^{q}(\mathrm{M}(\mathcal{A}), \mathbb{Z})$ is well studied (see e.g. [4), and especially, by Arnold-Brieskorn's result, it is generated by logarithmic forms

$$
\omega_{i}=\frac{1}{2 \pi \sqrt{-1}} \frac{d \alpha_{i}}{\alpha_{i}}
$$

for $i=1, \ldots, n$. The $q$-th cohomology group $H^{q}(\mathrm{M}(\mathcal{A}), \mathbb{Z})$ is spanned by $\omega_{i_{1}, \ldots, i_{q}}=$ $\omega_{i_{1}} \wedge \omega_{i_{2}} \wedge \cdots \wedge \omega_{i_{q}}$ with $H_{i_{1}}, \ldots, H_{i_{q}}$ linearly independent.

Theorem 5.1. Let $I=\left\{i_{1}, \ldots, i_{q}\right\} \subseteq\{1, \ldots, n\}$ be an ordered index (see $₫ 4.1$ for notation). Then

$$
\varphi\left(\omega_{I}\right)=(-1)^{q(\ell-q)} \varepsilon(I) \cdot \sum_{C}\left[\operatorname{cl}_{\mathrm{M}}(S(C))\right],
$$

where $C$ runs over all chambers $C \in \operatorname{ch}_{\mathcal{F}}^{q}(\mathcal{A})$ satisfying $C \subset C_{0}(I)$ and $\tau\left(X_{C}\right)=$ $\tau(X(I))$.

Proof. Let us define $S(I) \subset \mathbb{C}^{\ell}$ to be

$$
S(I)=C_{0}(I) \oplus \sqrt{-1} \cdot \tau(X(I)) .
$$

Then $\mathrm{cl}_{\mathrm{M}(\mathcal{A})}(S(I))$ is a disjoint union of $\operatorname{cl}_{\mathrm{M}}(S(C))$ 's with $C$ running over all chambers $C \in \operatorname{ch}_{\mathcal{F}}^{q}(\mathcal{A})$ satisfying $C \subset C_{0}(I)$ and $\tau\left(X_{C}\right)=\tau(X(I))$. It is enough to show that $\varphi\left(\omega_{I}\right)=(-1)^{q(\ell-q)} \varepsilon(I) \cdot \operatorname{cl}_{\mathrm{M}}(S(I))$. To do this, we shall consider the pairing with the homology class $\left[\sigma_{C^{\prime}}\right] \in H_{q}\left(\mathrm{M}^{q}, \mathrm{M}^{q-1}, \mathbb{Z}\right) \cong H_{q}(\mathrm{M}(\mathcal{A}), \mathbb{Z})$.

First we compute $\int_{\left[\sigma_{C^{\prime}}\right]} \omega_{I}$. The complement $\mathbb{C}^{\ell} \backslash \bigcup_{i \in I} H_{i, \mathbb{C}}$ is homotopy equivalent to $\left(\mathbb{C}^{*}\right)^{q} \simeq\left(S^{1}\right)^{q}$. The top homology $H_{q}\left(\mathbb{C}^{\ell} \backslash \bigcup_{i \in I} H_{i, \mathbb{C}}, \mathbb{Z}\right) \cong \mathbb{Z}$ is rank one. If $C^{\prime} \subset C_{0}(I)$, then $\left[\sigma_{C^{\prime}}\right]$ is transverse to $C_{0}(I)$. By applying Proposition 4.1 to the arrangement $\mathcal{A}(I)=\left\{H_{i_{1}}, \ldots, H_{i_{q}}\right\}$, we obtain the fact that $\left[\sigma_{C^{\prime}}\right]$ is a generator of $H_{q}\left(\mathbb{C}^{\ell} \backslash \bigcup_{i \in I} H_{i, \mathbb{C}}, \mathbb{Z}\right)$. Similarly, if $C^{\prime} \not \subset C_{0}(I)$, then $\left[\sigma_{C^{\prime}}\right]=0$. We have

$$
\int_{\left[\sigma_{C^{\prime}}\right]} \omega_{I}= \begin{cases}\varepsilon(I) & \text { if } C^{\prime} \subset C_{0}(I), \\ 0 & \text { else. }\end{cases}
$$


By a computation similar to the proof of Theorem 4.3, we have

$$
I\left([S(I)],\left[\sigma_{C^{\prime}}\right]\right)= \begin{cases}(-1)^{q(\ell-q)} & \text { if } C^{\prime} \subset C_{0}(I), \\ 0 & \text { else. }\end{cases}
$$

This completes the proof.

Remark 5.2. The correspondences between chambers and de Rham cohomology groups were investigated by Varchenko and Gel'fand in [9. Indeed, the cycle $S(I)$ appeared in their paper.

\section{REFERENCES}

1. A. Dimca, S. Papadima, Hypersurface complements, Milnor fibers and higher homotopy groups of arrangements. Ann. of Math. (2) 158 (2003), no. 2, 473-507. MR2018927 (2005a:32028)

2. M. Falk, Homotopy types of line arrangements. Invent. Math. 111 (1993), no. 1, 139-150. MR1193601 (93j:52020)

3. V. Guillemin, A. Pollack, Differential topology. Prentice-Hall, Inc., Englewood Cliffs, N.J., 1974. MR 0348781 (50:1276)

4. P. Orlik, L. Solomon, Combinatorics and topology of complements of hyperplanes. Invent. Math. 56 (1980), 167-189. MR.558866 (81e:32015)

5. P. Orlik, H. Terao, Arrangements of Hyperplanes. Grundlehren Math. Wiss. 300, SpringerVerlag, New York, 1992. MR1217488 (94e:52014)

6. R. Randell, Morse theory, Milnor fibers and minimality of hyperplane arrangements. Proc. Amer. Math. Soc. 130 (2002), no. 9, 2737-2743. MR.1900880(2003e:32048)

7. M. Salvetti, Topology of the complement of real hyperplanes in $C^{N}$. Invent. Math. 88 (1987), no. 3, 603-618. MR.884802 (88k:32038)

8. M. Salvetti, S. Settepanella, Combinatorial Morse theory and minimality of hyperplane arrangements. Geom. Topol. 11 (2007), 1733-1766. MR2350466 (2009c:32052)

9. A. Varchenko, I. M. Gel'fand, Heaviside functions of a configuration of hyperplanes. Functional Anal. Appl. 21 (1987), no. 4, 255-270 MR.925069 (89h:32028)

10. M. Yoshinaga, Hyperplane arrangements and Lefschetz's hyperplane section theorem. Kodai Math. J. 30 (2007) no. 2, 157-194. MR2343416 (2008i:32042)

11. M. Yoshinaga, The chamber basis of the Orlik-Solomon algebra and Aomoto complex. Arkiv för Matematik 47 (2009), 393-407. MR2529708 (2011a:32045)

12. T. Zaslavsky, Facing up to arrangements: Face-count formulas for partitions of space by hyperplanes. Memoirs Amer. Math. Soc. 154, 1975. MR0357135 (50:9603)

Research Institute for Mathematical Sciences, Kyoto University, Sakyo-ku, Kyoto 606-8502, JAPAN

E-mail address: koki@kurims.kyoto-u.ac.jp

Department of Mathematics, Faculty of Science, Kyoto University, Sakyo-ku, Kyoto 606-8502, JAPAN

E-mail address: mhyo@math.kyoto-u.ac.jp 